\title{
3 Research Square
Identifying Key Factors Associated with \\ Subscapularis Tendon Tears and Developing a Risk Prediction Model to Assist Diagnosis
}

\section{Wennan xu}

Orthopaedics Department, Beijing Hospital『National Center of Gerontology, Institute of Geriatric Medicine, Chinese Academy of Medical Sciences. Graduate School of Peking Union Medical College, P.R. $\mathrm{Ch}$

\section{Fei wang}

Orthopaedics Department, Beijing Hospital『National Center of Gerontology, Institute of Geriatric Medicine, Chinese Academy of Medical Sciences. Graduate School of Peking Union Medical College, P.R. $\mathrm{Ch}$

\section{Qingyun xue ( $\nabla$ xqybjhsp@163.com )}

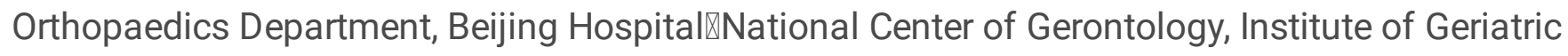
Medicine, Chinese Academy of Medical Sciences. Graduate School of Peking Union Medical College, P.R. $\mathrm{Ch}$

\section{Research Article}

Keywords: subscapularis, nomogram, tear, prediction

Posted Date: October 26th, 2021

DOI: https://doi.org/10.21203/rs.3.rs-1004157/v1

License: (c) (i) This work is licensed under a Creative Commons Attribution 4.0 International License. Read Full License 


\section{Abstract}

Background: Subscapularis (SSC) tendon tears can cause pain and restricted motion of the shoulder, but accurate diagnosis of this lesion on magnetic resonance imaging (MRI) is challenging, especially in small and partial tears. There are no studies that have established a method to reliably assess the risk of subscapularis tendon tears.

Methods: Data on 460 patients who received shoulder arthroscopic surgery with preoperative shoulder MRI were collected retrospectively. Of these, patients with SSC tendon tears were defined as the SSC tear group, and patients with intact subscapularis tendon were enrolled in the non-SSC tear group. Logistic regression analysis was used to identify the risk factors of SSC tendon tears which were then incorporated into the nomogram.

Results: Among 22 candidate factors, five independent factors including coracohumeral distance CHD (oblique sagittal) (OR, 0.75; 95\% Cl, [0.67-0.84]), fluid accumulation ( $\mathrm{Y}$-face) (OR, 2.29; 95\% $\mathrm{Cl},[1.20-4.38]$ ), long head of biceps tendon (LHB) dislocation/subluxation (OR, 3.62; 95\% Cl, [1.96-6.68]), number of posterosuperior (PS) rotator cuff tears (OR, 5.36; 95\% Cl, [3.12-9.22]), and MRI diagnosis (OR, 1.88; 95\% $\mathrm{Cl}$, [1.06-3.32]) were identified as key predictors associated with subscapularis tendon tears. Incorporating these predictors, the nomogram achieved a good $\mathrm{C}$ index with a good agreement on the risk estimation of calibration plots. Higher total points of the nomogram were associated with a greater risk of subscapularis tendon tears.

\section{Conclusion:}

The diagnostic accuracy of conventional 3.0-T MRI for SSC tendon tears was insufficient. Our study revealed critical predictors associated with subscapularis tendon tears. When evaluating the severity of subscapularis tendon injury, more attention should be paid to these tear-related factors. We developed and validated $s$ satisfactory prediction model to improve the diagnostic performance of MRI which was convenient for clinicians to reach a consensus on risk assessment and identify the SSC tendon tears.

\section{Introduction}

Subscapularis tendon was the largest rotator cuff tendon which plays an important role in maintaining the stability of the shoulder and internal rotation. Approximately $12-50 \%$ of patients were reported to have subscapularis tendon tears undergoing arthroscopy [1-3]. Lesions involving the subscapularis tendon can lead to pain and restricted motion of the shoulder. MRI plays an important role in diagnosing shoulder injuries because of its noninvasive and effectiveness. Although they provided good diagnostic performance in supraspinatus and infraspinatus tendon tears, the accurate diagnosis of subscapularis tendon tears is still unsatisfactory[4-6].

A systematic review and meta-analysis demonstrated that the overall sensitivity of MRI in the diagnosis of subscapularis tendon tears was only $68 \%[7]$. The width and thickness of subscapularis tendon tears 
directly affected the diagnostic performance of MRI. The smaller the tear size, the lower the accuracy of diagnosis, especially when the tear involved the superior one-third tendon[8]. Clinicians often directly evaluated the severity of subscapularis tendon injuries on MRI according to tendon continuity and signal changes, but there were still some limitations $[6,9,10]$. The diagnostic accuracy of subscapularis tendon tears was more dependent on their experience, which was various between clinicians[11]. We did not know how much assurance can support the accuracy of our suspicion based on experience alone, which directly affected the accuracy of our diagnosis and clinical decision. It was insufficient to rely on clinical and imaging experience to determine whether the accuracy of our diagnosis was consistent with the actual risk of SSC tendon tears.

Although several studies have investigated the risk factors associated with subscapularis tendon tears, research results remained controversial[12-15]. It is unclear which are the critical risk factors and the diagnostic value of these factors in predicting subscapularis tendon tears. We still lack an effective tool to evaluate the risk of subscapularis tendon tears. It is crucial to improve the identification of subscapularis tendon tears on MRI. We hope to reveal the key predictors and develop a novel measurement to convert this speculative experience to scientific risk estimation to assist the diagnosis.

Based on these reasons, the development of a prediction model that incorporates reliable factors associated with subscapularis tendon tears becomes desirable. Of all the available models, a nomogram can provide an evidence-based, individualized, and highly accurate risk estimation. Besides, nomogram is easy to use and can facilitate management-related decision-making. We sought to determine crucial predictors and establish a nomogram to predict SSC tendon tears. This study hypothesized that subscapularis tendon tears might be accurately identified by the risk prediction model.

\section{Methods}

\section{Patients}

This study was approved by our institutional review board (2021BJYYEC-225-01). The requirement for patient consent was waived by the review board because of the retrospective nature of the study. We retrospectively reviewed patients with shoulder arthroscopic surgery between January 2017, to December 2020. The inclusion criteria were patients who underwent shoulder arthroscopic procedures with preoperative shoulder MRI. Participants combined with fractures, tumors, immunologically related diseases, rheumatic immune diseases, and revision surgery of the shoulder were excluded.

Characteristics of patients included age, gender, cause of injury, injury side (i.e., left or right side). A total of 460 patients with 150 males and 310 females enrolled in the study. According to the Lafosse classification, we evaluated the severity of subscapularis tendon tears under arthroscopy[16]. Patients with arthroscopically determined subscapularis tendon tears were included in the SSC tear group, while others with intact subscapularis tendon were enrolled in the non-SSC tear group. Ultimately, 184 patients and 276 patients were included in the SSC tear group and non-SSC tear group respectively. The mean age 
of both groups was $62.55 \pm 9.03$ and $59.76 \pm 9.42$ years, respectively. Eighty-eight $(47.8 \%)$ patients in the SSC tear group underwent arthroscopic surgery for trauma, compared to $139(50.4 \%)$ in non-SSC tear group. A detailed description of characteristics was presented in table 1.

\section{Imaging characteristics}

All patients enrolled in the study received the identical imaging protocol. T1-T2-weighted and fatsuppressed T2-weighted images were performed underwent 3.0-T MRI with the arm in a neutral position. According to previous reports[14, 17-21], clinical experience, and clinical importance, seventeen imaging features were measured for further evaluation by two trained executors. The consensus was reached after deliberation and the average value of variables was obtained with multiple measurements.

Coracohumeral distance (CHD) was measured from the humeral cortex to the coracoid process cortex[17]. According to different measurement planes, we evaluated CHD on the oblique sagittal plane and axial plane, respectively (Figure.1). The coracoid overlap (CO) was defined as the distance between the glenoid and the tip of the coracoid process, which was measured on the axial plane[17] (Figure. 1). According to the previous study[18], the relative ratio of the coracoid length and humeral head diameter measured on the axial plane was defined as coracohumeral index (CHI) (Figure.1). To evaluate subscapularis tendon tears, Shim et al introduced two selected oblique sagittal planes (the en-face and $\mathrm{Y}$ face)[20]. The en-face plane was the image in which the glenoid was the largest observed and the base of the coracoid process was in contact with the glenoid, and the Y-view was the first image medial to the glenoid where the scapular spine was in contact with the scapular body (Figure. 2). We evaluated subscapular muscle atrophy and fluid accumulation on these two planes (Figure 2). In the en-face and the Y-face, subscapular muscle atrophy was classified as grades I, II, and III according to the degree of atrophy. In en-face, the atrophy of subscapularis was evaluated according to the base-to-tip line (BTL) introduced by Shim et al[20]. In Y -face, the atrophy of subscapularis was graded based on the tangent line and its parallel line. A detailed description of classification in subscapular muscle atrophy was presented in figure 2. To further evaluate fluid accumulation on en-face and Y-face, we introduced a new index, namely fluid area ratio. According to the base-to-tip line (BTL), the fluid area ratio was defined as the ratio of the effusion area to the area surrounded by the coracoid process, glenoid, and BTL in the enface (Figure.2). To facilitate the measurement of the fluid area ratio (en-face) on MRI, this indicator was

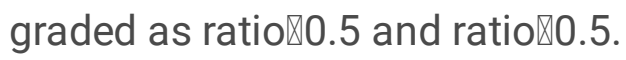

The posterosuperior (PS, supraspinatus, infraspinatus, teres minor) rotator cuff is anatomically adjacent to the subscapularis tendon. The retraction and severity of PS rotator cuff tears may affect the development and progression of subscapularis tendon lesions. To further investigate their relationship, the retraction of PS rotator cuff tears was classified according to the Patte classification (grade I III)[20, 22]. Furthermore, the severity of PS rotator cuff tears was classified on the basis of tearing thickness. Similarly, when a subscapularis tendon tear occurs due to the proximity of the anatomy, it is sometimes accompanied by a lesion of long head of the biceps (LHB)[14]. The malposition of LHB might be 
associated with subscapularis tendon tears. We selected LHB subluxation/dislocation as a potential risk factor with evaluation on the axial plane of MRI[23](Figure. 1). Turkmen et al suggested that the prevalence of subscapularis tendon tears was higher in patients with subcoracoid cyst[22] (Figure.1). In this study, the subcoracoid cyst was measured on axial plane with fat-suppressed T2-weighted MRI. Mostly, the greater tubercle cysts were considered to be associated with supraspinatus tenon tears[24, 25], clinicians speculated that the lesser tuberosity cysts (LTC) maybe be related to subscapularis tendon tears[15, 21]. We evaluated the presence of lesser tuberosity cysts and measured their maximum diameter on the fat-suppressed T2-weighted axial plane.

\section{Statistical Analysis}

Categorical variables were described as the whole number, and continuous variables were expressed as means \pm standard deviation. The significance of each variable in the cohort was assessed by univariable logistic regression analysis firstly. Variables significantly associated with subscapularis tendon tears were further assessed in multivariable logistic regression (forward stepwise likelihood ratio method) to select the final independent risk factors.

Continuous variables (ie, age and CHD) were directly analyzed in the logistic regression, while for ordered categorical variables, we converted them into dichotomous variables with optimal scale regression before entering logistic regression. P-value $<0.05$ was considered to be statistically significant.

According to the results of multivariable logistic regression, the rms package of $\mathrm{R}$, version 4.0 (http://www.r-project.org/) was used to establish a nomogram. The nomogram was based on proportionally converting each regression coefficient in multivariate logistic regression to a 0 - to 100-point scale. The total points were derived from the sum of the points for each independent variable and converted into predicted probability. The predictive performance of the nomogram is evaluated by concordance index (C index), decision curve analysis (DCA), and calibration with 1000 bootstrap samples. The performance of the predictive model was evaluated by the sensitivity, specificity, predictive values, and likelihood ratios.

\section{Results}

\section{Clinical, imaging, and arthroscopic characteristics.}

A total of 460 patients met the inclusion criteria and the characteristics were presented in table 1. According to clinical importance, scientific knowledge, and previous reports, a total of 22 variables were selected as candidates. Continuous variables including age, $\mathrm{CHD}, \mathrm{CO}$, and $\mathrm{CHI}$ were analyzed directly with logistic regression analysis.

Dichotomous variables such as gender, cause of injury, injured side, fluid accumulation, subcoracoid cyst, LHB dislocation/subluxation, and the number of PS rotator cuff tears were also analyzed by logistic 
regression analysis. In SSC tear group, 154 (83.7\%), 58 (31.5\%), and 122ه66.3\%囚patients with fluid accumulation were identified in the En-face, Y-face, and coronal plane, respectively, compared with 191 (69.2\%), 23 (8.3\%), and 140 (50.7\%) patients in non-SSC tear group. In SSC tear group eighty-eight (47.8\%) patients received shoulder arthroscopic procedures due to trauma compared with 139 patients (50.4\%) in non-SSC tear group. Subcoracoid cysts were determined in 106 (57.6\%) and $73(26.4 \%)$ patients in the SSC tear group and non-SSC tear group, respectively. In patients with SSC tendon tears, 70 $(38.0 \%)$ of them were accompanied by LHB dislocated/subluxation, whereas in the non-SSC tear group, $24(8.7 \%)$ patients had a combined LHB dislocation/subluxation. Ninety $(48.9 \%)$ patients with SSC tendon tears combined with multiple PS rotator cuff tears, while in non-SSC tear group, only $28(10.1 \%)$ patients had multiple PS rotator cuff tears. In addition, we also presented the result of MRI diagnosis in subscapularis tendon tears.

Ordered categorical variables such as atrophy, patte classification, and thickness of tear were converted into dichotomous variables according to the inflection point with the most obvious change through optimal scale regression. We converted muscle atrophy to a dichotomous variable and classified it as grade I and grade II/III. Similarly, patte classification was converted to normal/grade I and grade II/III, and the thickness of PS rotator cuff tear was divided into full-thickness tear and non full-thickness tear (partial and no tear).

\section{Features selection and determination}

After definition, conversion, and classification, univariable logistic regression was used to evaluate these 22 characteristics. The results of univariable logistic regrssion are presented in table 1. In univariable logistic regression, significant differences were found in 16 variables which were further evaluated by multivariable logistic regression. Variables for inclusion were carefully chosen, given the number of events available, to ensure parsimony of the final model. The forward stepwise likelihood ratio method was used in the multivariable logistic regression to identify the most related independent risk factors of subscapularis tendon tears. All of the independent predictors were reported as odds ratios $(95 \% \mathrm{Cl})$. Eventually, $\mathrm{CHD}$ (oblique sagittal) $(\mathrm{OR}, 0.75 ; 95 \% \mathrm{Cl},[0.67-0.84])$, fluid accumulation (Y-face) (OR, 2.29; $95 \% \mathrm{Cl}$, [1.20-4.38]), LHB dislocation/subluxation (OR, 3.62; 95\% Cl, [1.96-6.68]), number of PS rotator cuff tears $(\mathrm{OR}, 5.36 ; 95 \% \mathrm{Cl}$, [3.12-9.22]), and MRI diagnosis (OR, 1.88; 95\% Cl, [1.06-3.32]) were significantly associated with subscapularis tendon tears (Table. 2).

\section{Nomogram and Model Performance}

A Nomogram to predict subscapularis tendon tears based on these 5 related risk factors was shown in figure 3. The generated model was internally validated with the 1000 bootstrap validation method. This nomogram demonstrated good discriminative ability in estimating the risk of SSC tendon tears with a C index of 0.820 (0.782-0.858). In addition, calibration plots graphically showed good agreement on the risk estimation by the nomogram with 1000 resampling (Figure.3). Patients with decreased CHD (oblique 
sagittal), LHB dislocation/subluxation, fluid accumulation (Y-face), multiple PS rotator cuff tears, and MRI diagnosis of SSC tendon tears were associated with higher nomogram points. Higher total points based on the sum of points for independent variables in the nomogram were associated with a greater risk subscapularis tendon tears.

For comparison with the diagnostic performance of MRI in diagnosing subscapularis tendon tears, we calculated the area under the receiver-operating characteristic (ROC) curve for these two methods (Figure.4). The sensitivity, specificity, positive predictive value, negative predictive value, positive likelihood ratio, and negative likelihood ratio were shown in table 3. In addition, we also performed decision curve analysis (DCA) for assessment of the clinical utility in these two methods (Figure 4).

\section{Discussion}

The most important contribution of this study was that we identified critical predictors among numerous factors and determined their clinical importance in the diagnosis of subscapularis tendon tears. Patients with decreased CHD (oblique sagittal), fluid accumulation (Y-face), LHB dislocation/subluxation, multiple PS rotator cuff tears, and MRI diagnosis of SSC tendon tears were accompanied by a higher risk of subscapularis tendon tears. When evaluating subscapularis tendon injuries, clinicians should attach importance to the diagnostic value of these predictors. Beyond that, the study developed and validated a nomogram that provided reliable risk estimation of SSC tendon tears, which could assist clinicians in diagnosis. When evaluating subscapularis tendon tears, this prediction model could improve the diagnostic performance of conventional MRI.

Many scholars implied that the morphology of the coracoid process could affect the development and progression of subscapularis tendinopathy $[17,18,26]$. They suggested that $\mathrm{CHD}, \mathrm{CO}$, and $\mathrm{CHI}$ were potential predictors in SSC tendon tears[17, 18, 27], although others presented different insights[12, 28]. To further evaluate the relationship between coracoid morphology and subscapularis tendon tears, we measured $\mathrm{CHD}$ on axial and oblique sagittal planes respectively. The result indicated that a decreased CHD (oblique sagittal plane) was significantly associated with a greater risk of SSC tendon tears. But, we did not find any significant correlation of $\mathrm{CHI}$ and $\mathrm{CO}$ in subscapularis tendon tears.

Anatomically, the subscapularis tendon is interdependent with the PS rotator cuff and LHB to support the biceps pully structure. Some scholars suggested that the lesion of PS rotator cuff and LHB were associated with subscapularis tendon tears $[13,14,29]$. In our study, the result of multivariable logistic regression indicated that LHB subluxations/dislocation and multiple PS rotator cuff tears were significantly associated with SSC tendon tears. Patients with these risk factors were prone to acquiring subscapularis tendon tears.

Previous studies attempted to reveal the relationship between fluid signal and subscapularis tendon tears[15, 20, 22]. They suggested that patients with subcoracoid cyst, lesser tuberosity cyst, and fluid accumulation around the subscapularis were more likely to have subscapularis tendon tears. In this study, we introduced a new index, namely the fluid area ratio. To facilitate the evaluation of this indicator 
on MRI we divided the fluid area ratio into two-level, namely ratio $₫ 0.5$ and ratio $₫ 0.5$. Despite we did not find lesser tuberosity cyst, fluid area ratio, and subcoracoid cyst were associated with SSC tendon tears, the result recommended fluid accumulation ( $\mathrm{Y}$-face) as a strong predictor of SSC tendon tears. Patients with a fluid accumulation of Y-face on MRI indicated a higher risk of subscapularis tendon tears.

Eventually, CHD (oblique sagittal), fluid accumulation (Y-face), LHB dislocation/subluxation, number of PS rotator cuff tears, and MRI diagnosis of SSC tendon tears were determined as critical predictors associated with subscapularis tendon tears. The current study provided new insight into evaluating subscapularis tendon tears. The ROC and DCA curves showed that the nomogram based on the imaging characteristics has better predictive ability and higher clinical usefulness than conventional MRI. This risk prediction model could achieve superior diagnostic performance in SSC tendon tears, which is helpful to reduce the missed diagnosis and assist in making clinical decisions.

As a new predictive tool based on reliable predictors, this nomogram was convenient to reach a consensus on risk assessment to assist clinicians in diagnosis. But there were still some limitations in our study. First, this analysis was based on the data of a single institution, it is necessary to validate the results from other centers (external validation) in the future. Second, although physical examination tests are routine procedures, we did not include them in this study because of their susceptibility to physicians and patients' subjectivity and the limited accuracy reported in the previous literature[30]. In future studies, we will investigate reliable clinical characteristics to further improve the performance of this risk prediction model in the evaluation of subscapularis tendon tears.

\section{Conclusion}

Conventional MRI was insufficient to accurately diagnosis subscapularis tendon tears based on the continuity and signal changes of the tendon. This nomogram provided a satisfactory prediction of SSC tendon tears which could assist clinicians in diagnosis. When evaluating the severity of subscapularis tendon injuries, we should give increasing attention to these tear-related features. Using this prediction model could improve the diagnostic performance of conventional MRI which is convenient for clinicians to reach a consensus on risk assessment of SSC tendon tears and identify patients who may need further arthroscopy.

\section{Abbreviations}

coracohumeral distance : CHD

coracoid overlap : CO

coracohumeral index: $\mathrm{CHI}$

receiver-operating characteristic : ROC 
decision curve analysis : DCA

long head of the biceps :LHB

lesser tuberosity cyst: LTC

subscapularis : SSC

magnetic resonance imaging: MRI

odds ration: $\mathrm{OR}$

posterosuperior : PS

\section{Declarations}

\section{Ethics approval and consent to participate}

This study was performed in accordance with the Declaration of Helsinki principles and was approved by the Institutional Review Board of Beijing Hospital(2021BJYYEC-225-01). The requirement for patient consent was waived by the review board because of the retrospective nature of the study.

\section{Consent for publication}

Not applicable.

\section{Availability of data and materials}

The datasets used and/or analysed during the current study are available from the corresponding author on reasonable request.

\section{Competing interests}

The authors declare that they have no competing interests.

\section{Funding}

Not applicable.

\section{Authors' contributions}


All authors contributed to the study conception and design as well as the material preparation, data collection and analysis. The first draft of the manuscript was written by Wennan xu and all authors commented on previous versions of the manuscript. All authors read and approved the final manuscript.

\section{Acknowledgements}

Not applicable.

\section{References}

1. Waldt S, Bruegel M, Mueller D, et al. Rotator cuff tears: assessment with MR arthrography in 275 patients with arthroscopic correlation. Eur Radiol.Feb 2007;17(2):491-498.

2. Lee JH, Yoon YC, Jee S, Kwon JW, Cha JG, Yoo JC. Comparison of three-dimensional isotropic and two-dimensional conventional indirect MR arthrography for the diagnosis of rotator cuff tears. Korean J Radiol.Nov-Dec 2014;15(6):771-780.

3. Narasimhan R, Shamse K, Nash C, Dhingra D, Kennedy S. Prevalence of subscapularis tears and accuracy of shoulder ultrasound in pre-operative diagnosis. Int Orthop.May 2016;40(5):975-979.

4. Lin L, Yan H, Xiao J, et al. The diagnostic value of magnetic resonance imaging for different types of subscapularis lesions. Knee Surg Sports Traumatol Arthrosc.Jul 2016;24(7):2252-2258.

5. Sela Y, Eshed I, Shapira S, et al. Rotator cuff tears: correlation between geometric tear patterns on MRI and arthroscopy and pre- and postoperative clinical findings. Acta Radiol.Feb 2015;56(2):182189.

6. Ward JRN, Lotfi N, Dias RG, McBride TJ. Diagnostic difficulties in the radiological assessment of subscapularis tears. J Orthop.Mar 2018;15(1):99-101.

7. Malavolta EA, Assunção JH, Gracitelli MEC, Yen TK, Bordalo-Rodrigues M, Ferreira Neto AA. Accuracy of magnetic resonance imaging (MRI) for subscapularis tear: a systematic review and meta-analysis of diagnostic studies. Arch Orthop Trauma Surg.May 2019;139(5):659-667.

8. Naimark M, Zhang AL, Leon I, Trivellas A, Feeley BT, Ma CB. Clinical, Radiographic, and Surgical Presentation of Subscapularis Tendon Tears: A Retrospective Analysis of 139 Patients. Arthroscopy.May 2016;32(5):747-752.

9. Banerjee M, Müller-Hübenthal J, Grimme S, et al. Moderate value of non-contrast magnetic resonance imaging after non-dislocating shoulder trauma. Knee Surg Sports Traumatol Arthrosc.Jun 2016;24(6):1888-1895.

10. Ramadan LB, Baptista E, Souza FF, et al. Diagnostic accuracy of preoperative magnetic resonance imaging for detecting subscapularis tendon tears: a diagnostic test study. Sao Paulo Med J.Jul-Aug 2020;138(4):310-316.

11. Ryu HY, Song SY, Yoo JC, Yun JY, Yoon YC. Accuracy of sagittal oblique view in preoperative indirect magnetic resonance arthrography for diagnosis of tears involving the upper third of the 
subscapularis tendon. J Shoulder Elbow Surg.Dec 2016;25(12):1944-1953.

12. Cetinkaya M, Ataoglu MB, Ozer M, Ayanoglu T, Kanatli U. Subscapularis Tendon Slip Number and Coracoid Overlap Are More Related Parameters for Subcoracoid Impingement in Subscapularis Tears: A Magnetic Resonance Imaging Comparison Study. Arthroscopy.Apr 2017;33(4):734-742.

13. Mehta SK, Teefey SA, Middleton W, Steger-May K, Sefko JA, Keener JD. Prevalence and risk factors for development of subscapularis and biceps pathology in shoulders with degenerative rotator cuff disease: a prospective cohort evaluation. J Shoulder Elbow Surg.Mar 2020;29(3):451-458.

14. Yoon JS, Kim SJ, Choi YR, Lee W, Kim SH, Chun YM. Medial Subluxation or Dislocation of the Biceps on Magnetic Resonance Arthrography Is Reliably Correlated with Concurrent Subscapularis FullThickness Tears Confirmed Arthroscopically. Biomed Res Int.2018;2018:2674061.

15. Cetinkaya M, Öner AY, Ataoglu MB, Ozer M, Ayanoglu T, Kanatli U. Lesser tuberosity cysts and their relationship with subscapularis tears and subcoracoid impingement. J Orthop Sci.Jan 2017;22(1):6368.

16. Lafosse L, Jost B, Reiland Y, Audebert S, Toussaint B, Gobezie R. Structural integrity and clinical outcomes after arthroscopic repair of isolated subscapularis tears. J Bone Joint Surg Am.Jun 2007;89(6):1184-1193.

17. Leite MJ, Sá MC, Lopes MJ, Matos RM, Sousa AN, Torres JM. Coracohumeral distance and coracoid overlap as predictors of subscapularis and long head of the biceps injuries. J Shoulder Elbow Surg.Sep 2019;28(9):1723-1727.

18. Zhang H, Zhang Q, Li ZL. Coracohumeral index and coracoglenoid inclination as predictors for different types of degenerative subscapularis tendon tears. Int Orthop.Aug 2019;43(8):1909-1916.

19. Meyer DC, Zimmermann SM, Wieser K, Bensler S, Gerber C, Germann M. Lengthening of the subscapularis tendon as a sign of partial tearing in continuity. J Shoulder Elbow Surg.Jan 2016;25(1):31-37.

20. Shim JW, Pang CH, Min SK, Jeong JY, Yoo JC. A novel diagnostic method to predict subscapularis tendon tear with sagittal oblique view magnetic resonance imaging. Knee Surg Sports Traumatol Arthrosc.Jan 2019;27(1):277-288.

21. Wissman RD, Ingalls J, Hendry D, Gorman D, Kenter K. Cysts within and adjacent to the lesser tuberosity: correlation with shoulder arthroscopy. Skeletal Radiol.Sep 2012;41(9):1105-1110.

22. Turkmen I, Altun G, Celik H, Bilsel K. Can subcoracoid cyst formation be a sign of anterosuperior rotator cuff tears and biceps pulley lesions? A prospective radiologic and arthroscopic correlation study. J Shoulder Elbow Surg.Aug 2020;29(8):1665-1670.

23. Shi LL, Mullen MG, Freehill MT, Lin A, Warner JJ, Higgins LD. Accuracy of long head of the biceps subluxation as a predictor for subscapularis tears. Arthroscopy.Apr 2015;31(4):615-619.

24. Chillemi C, Paglialunga C, Guerrisi M, Mantovani M, Osimani M. Arthroscopic Transosseous Repair of Rotator Cuff Tear and Greater Tuberosity Cysts. Arthrosc Sports Med Rehabil.Jun 2020;2(3):e241e250. 
25. Chin K, Chowdhury A, Leivadiotou D, Marmery H, Ahrens PM. The accuracy of plain radiographs in diagnosing degenerate rotator cuff disease. Shoulder Elbow.May 2019;11(1 Suppl):46-51.

26. Hodax JD, Shah KN, Campbell SE, Cameron KL, Owens BD. Measurement of the coracohumeral distance on magnetic resonance imaging in a large patient cohort. J Shoulder Elbow Surg.Feb 2021;30(2):408-412.

27. Zhu S, Tan J, Wu D, Hu N, Huang W, Chen H. Bilateral coracohumeral distance discrepancy is associated with subscapularis tear in rotator cuff rupture patients. Knee Surg Sports Traumatol Arthrosc.May 62021.

28. Tollemar VC, Wang J, Koh JL, Lee MJ, Shi LL. Coracoid morphology is not associated with subscapularis tears. J Shoulder Elbow Surg.Jun 2020;29(6):1162-1167.

29. Kim BR, Lee J, Ahn JM, et al. Predicting the clinically significant subscapularis tendon tear: malposition and tear of the long head of the biceps tendon on shoulder magnetic resonance imaging. Acta Radiol.Dec 16 2020:284185120980017.

30. Gismervik S, Drogset JO, Granviken F, Rø M, Leivseth G. Physical examination tests of the shoulder: a systematic review and meta-analysis of diagnostic test performance. BMC Musculoskelet Disord.Jan 25 2017;18(1):41.

\section{Tables}

\section{Table 1}

characteristics and result of univariable logistic analysis 
Variable

Cohort

OR $(95 \% \mathrm{Cl})$

P
Value

SSC tear

non-SSC

tear

Age, year

$62.55 \pm$

9.03

$59.76 \pm$

9.42

$1.03(1.01-$

1.06)

Gender

male

female

Cause

degenerative

traumatic

Injured side

right

CHD (axial), mm

$\mathrm{CHD}$ (oblique sagittal), $\mathrm{mm}$

112

72

$8.04 \pm$

2.25

$8.17 \pm$

2.08

$\mathrm{cO}, \mathrm{mm}$

$\mathrm{CHI}$

$15.96 \pm$

3.84

$0.33 \pm$

0.08

Fluid accumulation (En-face)

$\begin{array}{lllll}\text { yes } & 154 & 191 & 2.28(1.43- & 0.001 \\ \text { no } & 30 & 85 & 3.65) & \end{array}$

Fluid area ratio (En-face), ratio $₫ 0.5$

$\begin{array}{lllll}\text { yes } & 30 & 12 & 4.29(2.13- & <0.001 \\ \text { no } & 154 & 264 & 8.62) & \end{array}$

Fluid accumulation (Y-face) yes
155

$1.21(0.83-$

1.78)

0.316

121

$9.28 \pm 2.46$

$0.79(0.72-$

$0.86)$

$<0.001$

$9.73 \pm 2.60$

0.75 (0.68-

$0.82)$

$<0.001$

$15.84 \pm$

3.94

$1.01(0.96-$

1.06)

0.743

$0.52 \pm 3.14$

$2.52(0.22-$

28.94)

0.457

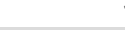


no

126

253

8.59)

Fluid accumulation (Coronal)

$\begin{array}{lllll}\text { yes } & 122 & 140 & 1.91(1.30- & 0.001 \\ \text { no } & 62 & 136 & 2.81) & \end{array}$

Atrophy (En-face)

grade I

grade II/III

Atrophy (Y-face)

grade I

grade II/III

Subcoracoid cyst

yes

no

Lesser tuberosity cyst, number $\geq 1$

$\begin{array}{lllll}\text { yes } & 62 & 70 & 1.47(0.98- & 0.065 \\ \text { no } & 122 & 206 & 2.21) & \end{array}$

Lesser tuberosity cyst, diameter $\geq 5$

$(\mathrm{mm})$

$\begin{array}{lllll}\text { yes } & 18 & 13 & 2.19(1.05- & 0.037 \\ \text { no } & 166 & 263 & 4.60) & \end{array}$

LHB dislocation/subluxation

$\begin{array}{lllll}\text { yes } & 70 & 24 & 6.45(3.86- & <0.001 \\ \text { no } & 114 & 252 & 10.78) & \end{array}$

Patte

$\begin{array}{lllll}\text { normal/grade I } & 120 & 246 & 4.37(2.69- & <0.001 \\ \text { grade II/III } & 64 & 30 & 7.11) & \end{array}$

Classification

non full-thickness tear

full-thickness tear

88

96
$5.04(2.89-$

$8.80)$

$2.65(1.75-\quad<0.001$ $4.00)$

$3.78(2.54-\quad<0.001$ $5.62)$

58

$106 \quad 73$

$78 \quad 203$




\begin{tabular}{ccccc}
\hline$\leq 1$ & 94 & 248 & $8.48(5.22-$ & $<0.001$ \\
\hline 2 & 90 & 28 & $13.79)$ & \\
\hline MRI diagnosis & & & & \\
\hline SSC tear & 56 & 43 & $2.37(1.51-$ & $<0.001$ \\
\hline non-SSC tear & 128 & 233 & $3.73)^{-}$ &
\end{tabular}

LHB long head of biceps tendon. SSC subscapularis. CHD coracohumeral distance. CO coracoid overlap. CHI coracohumeral index. OR odds ratio.

\section{Table. 2}

Result of multivariable logistic analysis

\begin{tabular}{|c|c|c|c|}
\hline Variable & $\beta$ & OR $(95 \% \mathrm{Cl})$ & P Value \\
\hline CHD (oblique sagittal), mm & -0.29 & $0.75(0.67-0.84)$ & $\otimes 0.001$ \\
\hline Fluid accumulation (Y-face) & 0.83 & $2.29(1.20-4.38)$ & 0.012 \\
\hline LHB (dislocation/subluxation) & 1.29 & $3.62(1.96-6.68)$ & $\otimes 0.001$ \\
\hline Number of tears $(\leq 1$ or $\geq 2)$ & 1.68 & $5.36(3.12-9.22)$ & $₫ 0.001$ \\
\hline MRI diagnosis & 0.63 & $1.88(1.06-3.32)$ & 0.03 \\
\hline
\end{tabular}

CHD coracohumeral distance. LHB long head of biceps tendon. OR Odds Ratio.

\section{Table. 3}

Diagnostic performance of nomogram and MRI for predicting subscapularis tendon tears. 
Variable

Value-nomogram $(95 \% \mathrm{Cl}) \quad$ Value-MRI $(95 \% \mathrm{Cl})$

Area under ROC curve $\quad 0.820(0.782-0.858) \quad 0.574(0.504-0.644)$

Sensitivity, \%

$82.6(76.2-87.6)$

$30.4(21.5-41.0)$

Specificity, \%

$65.6(59.6-71.1)$

$84.4(79.5-88.4)$

Positive predictive value, $\%$

$61.5(55.1-67.6)$

$39.4(28.3-51.8)$

Negative predictive value, $\% \quad 85.0$ (79.3-89.4)

$78.5(73.2-82.9)$

Positive likelihood ratio

$2.40(2.01-2.86)$

$1.95(1.29-2.95)$

Negative likelihood ratio

$0.27(0.19-0.36)$

$0.82(0.72-0.94)$

MRI magnetic resonance imaging. ROC receiver operating characteristic. $\mathrm{Cl}$ confidence interval.

\section{Figures}




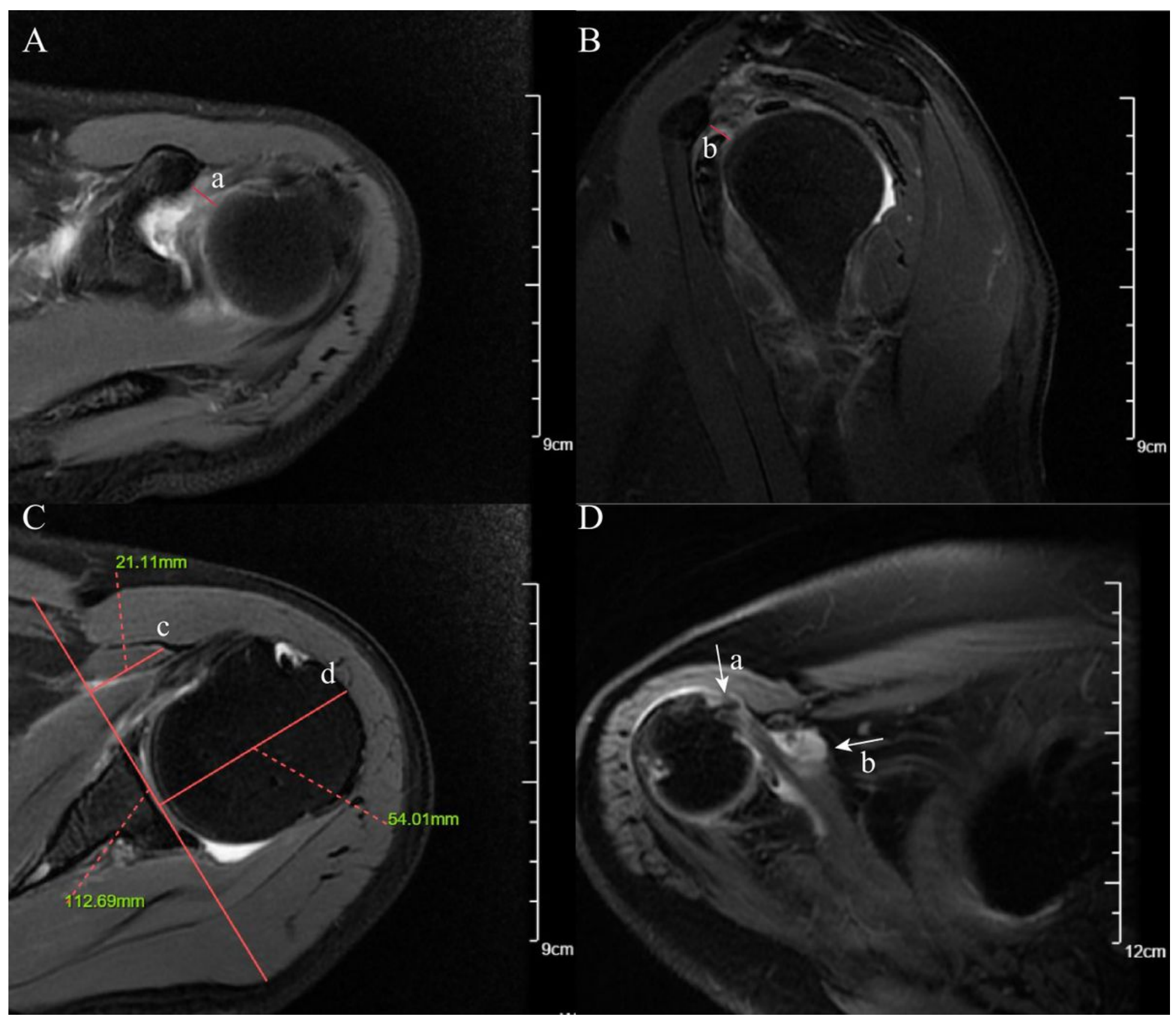

\section{Figure 1}

Fat-suppressed T2-weighted MRI images of coracohumeral distance (CHD), coracoid overlap (CO), coracohumeral index (CHI), LHB dislocation/subluxation and subcoracoid cyst. A CHD measured on axial plane (red solid line a). B CHD measured on oblique sagittal plane (red solid line b). C CO (red solid line c), $\mathrm{CHI}$ (red solid line $\mathrm{c} /$ red solid line $\mathrm{d}$, namely $21.11 \mathrm{~mm} / 54.01 \mathrm{~mm}$ ). D Subcoracoid cyst on axial plane (white arrow b). Dislocation/subluxation of LHB on axial plane (white arrow a). 


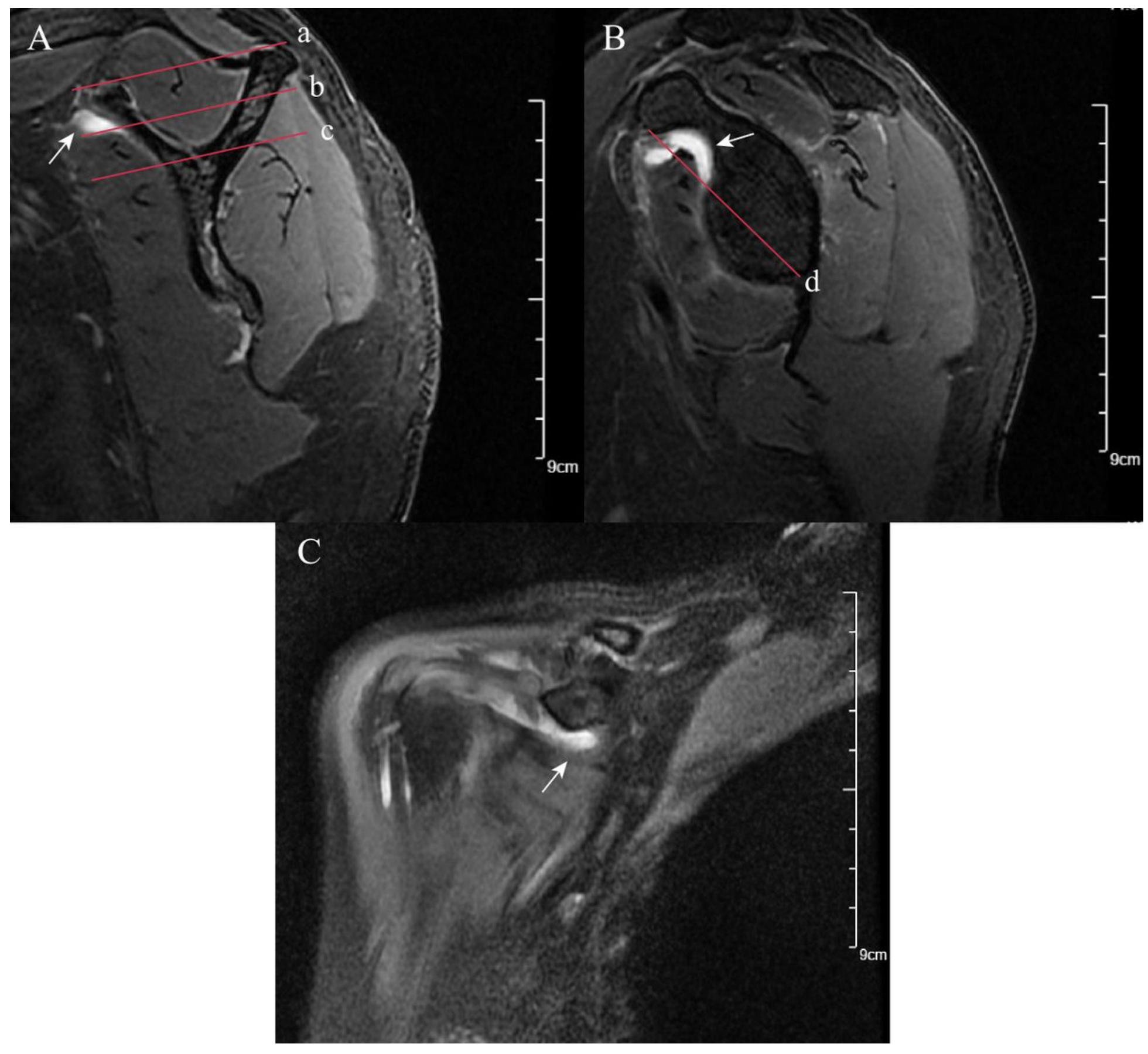

\section{Figure 2}

MRI images of subscapular muscle atrophy, fluid accumulation, and fluid area ratio (en-face). A SSC muscle atrophy classification on $\mathrm{Y}$ - face plane. Parallel line intercepting supraspinatus fossa opening (red solid line a), and parallel line (red solid line b) bisecting the perpendicular distance between line (red solid line a) and line (red solid line c). Based on these lines, subscapularis position was classified.

Between line (a) and (b) was classified as grade (I), between line (b) and (c) was classified as grade (II), below line (c) was classified as grade (III). Fluid accumulation in the $\mathrm{Y}$-face plane (white arrow). B En-face plane showing base to-tip line (BTL): the inferior pole of glenoid to coracoid tip on en-face (red solid line d). Using BTL, muscle atrophy of subscapularis is classified as grade I (tendon and muscle exist above the BTL), grade II (only tendon exist above the BTL), and grade III (tendon and muscle exist below the 
$B T L$ ). The fluid area ratio was the ratio of the effusion area to the area surrounded by the coracoid process, glenoid, and BTL in the en-face. Fluid accumulation in the en-face plane (white arrow). C Fluid accumulation in the coronal plane (white arrow).

A

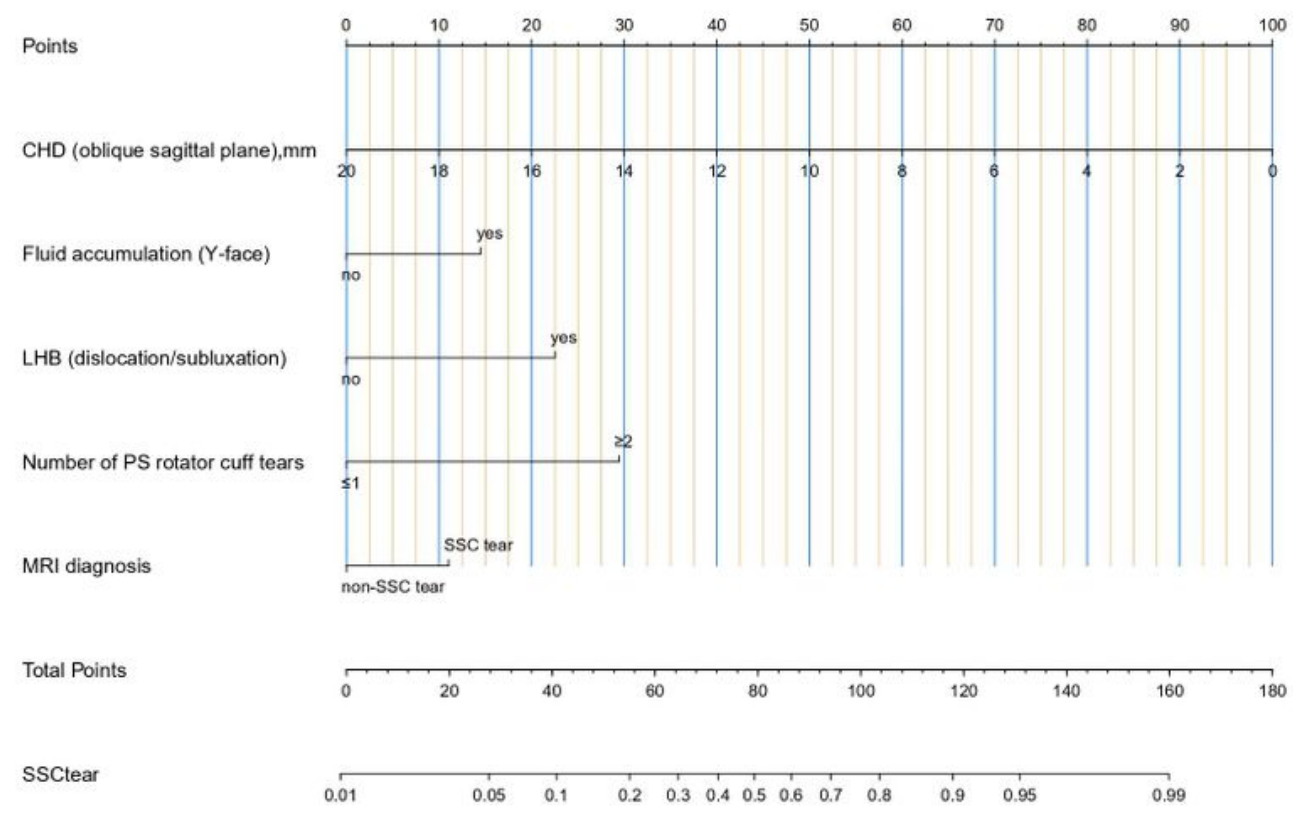

$\mathrm{B}$

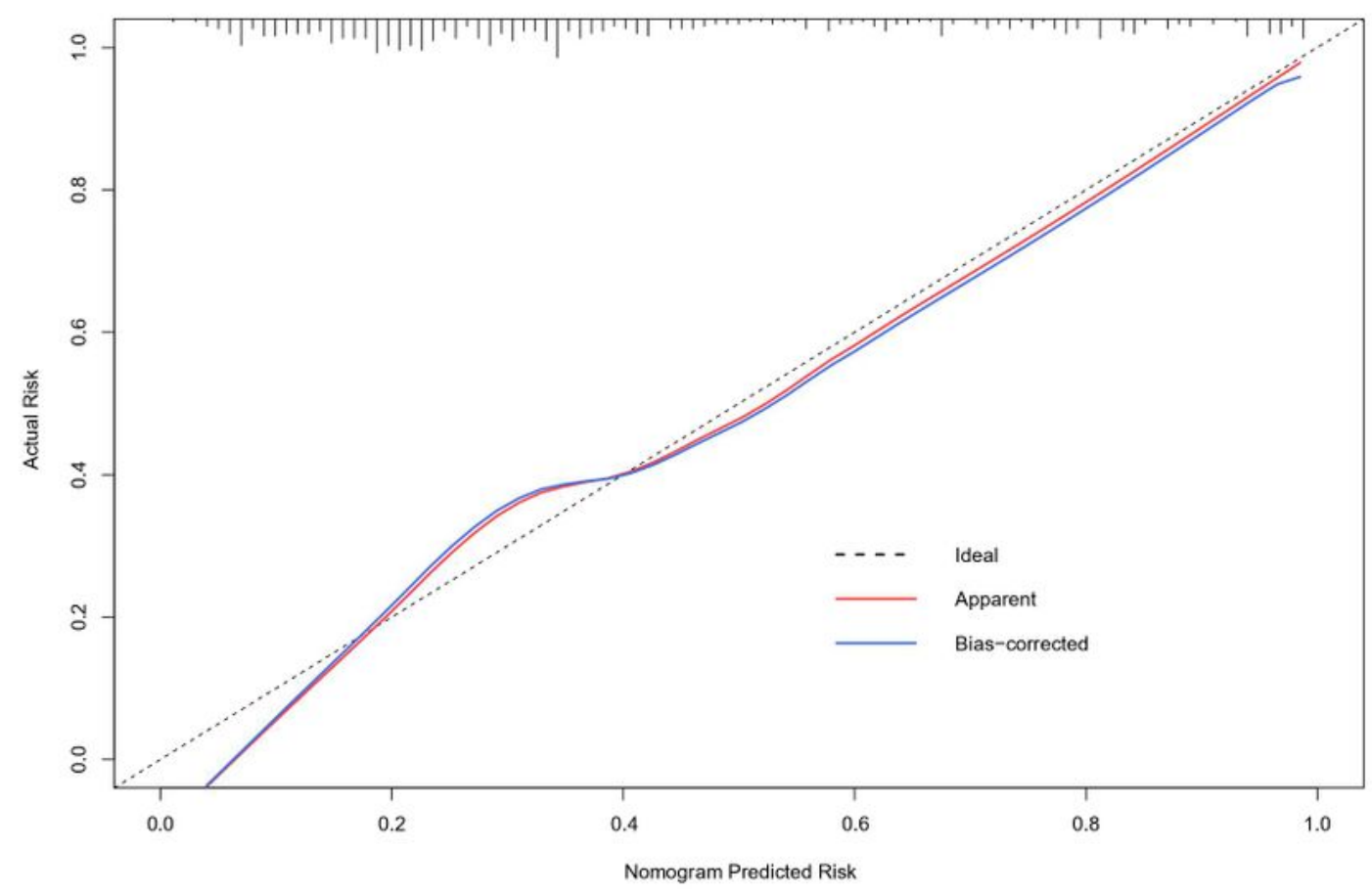

Figure 3

Nomogram and calibration. A Nomogram. To use the nomogram, find the position of each variable on the corresponding axis, draw a line to the points axis for the number of points, add the points from all of the 
variables, and draw a line from the total points axis to determine the tear risk of subscapularis tendon at the lower line of the nomogram. B Calibration plot. Validity of the predictive performance of the nomogram in estimating the risk of SSC tendon tears with the 1000-sample bootstrapped calibration plot. The calibration curve demonstrated that our nomograms have good predictive performance.

A

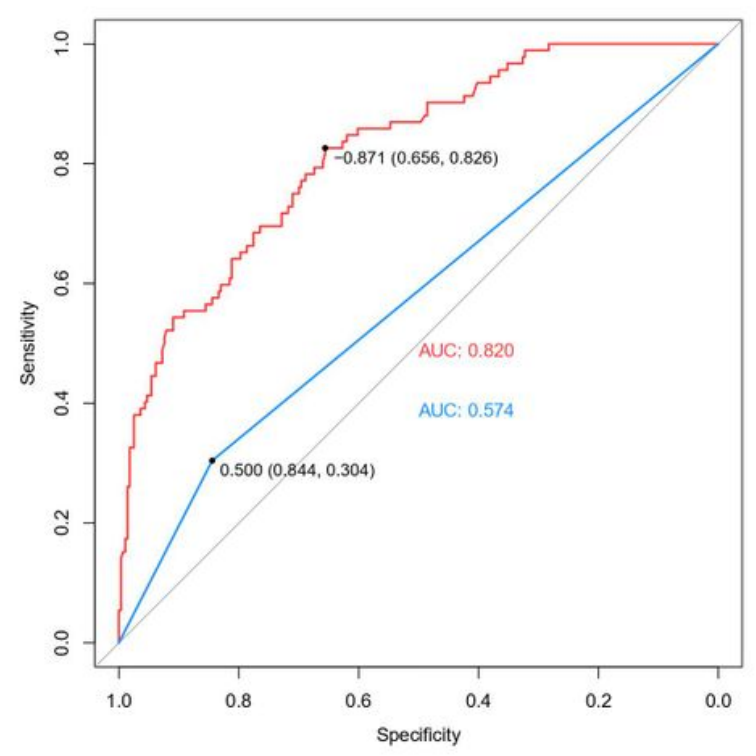

B

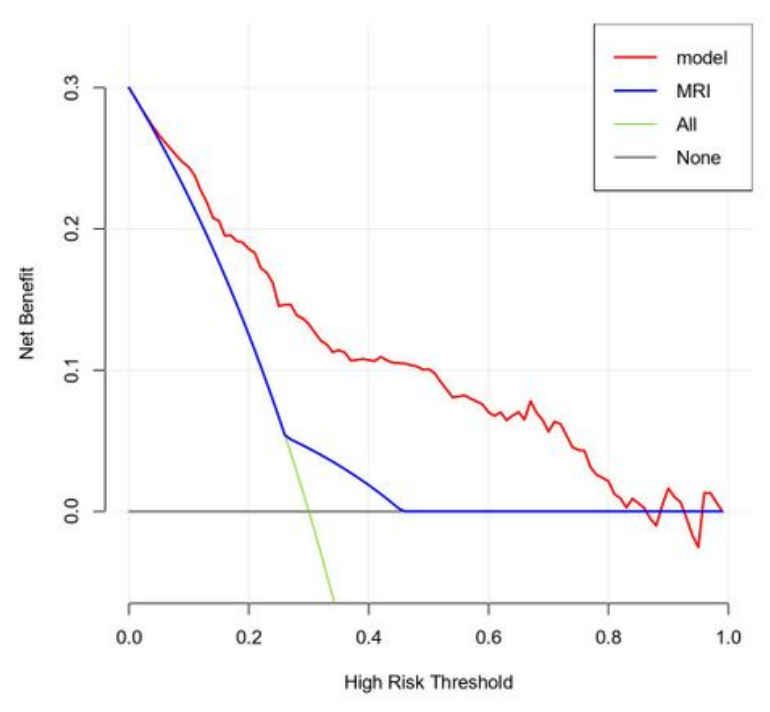

Figure 4

ROC and DCA curve. A AUC of two methods (nomogram : 0.820, MRI: 0.574). B DCA of two methods. DCA curve analysis showed this model (nomogram) have comparable clinical performance with higher clinical application value and better clinical practicability than MRI. 